\title{
Requirements for an Evaluation Infrastructure for Reliable Pervasive Healthcare Research
}

\author{
Stefan Wagner \\ Department of Engineering \\ Aarhus University \\ Finlandsgade 22, Aarhus, Denmark \\ sw@iha.dk
}

\author{
Thomas S. Toftegaard \\ Department of Engineering \\ Aarhus University \\ Finlandsgade 22, Aarhus, Denmark \\ tst@cs.au.dk
}

\author{
Olav W. Bertelsen \\ Department of Computer Science \\ Aarhus University \\ Aabogade 34, Aarhus, Denmark \\ olavb@cs.au.dk
}

\begin{abstract}
- the need for a non-intrusive evaluation infrastructure platform to support research on reliable pervasive healthcare in the unsupervised setting is analyzed and challenges and possibilities are identified. A list of requirements is presented and a solution is suggested that would allow researchers to more easily build and evaluate prototypes for measuring and quantifying the use-context of patients using current state-of-theart biomedical devices in the unsupervised setting. An initial implementation is introduced as the reliable evaluation infrastructure (RELEI) platform. Several research prototypes using the basic RELEI platform are presented to illustrate the purpose, and provide experiences on platform usage.
\end{abstract}

Keywords-component; reliability, pervasive healthcare, pervasive computing, context-aware, adherence, compliance.

\section{INTRODUCTION}

Healthcare systems are challenged by the demographic changes of the coming decades and the resulting projected massive increase in chronic patient group levels [1-3]. Many studies have investigated the potential of moving patient care out of the hospitals and clinics, and into the home setting, increasing patient-empowerment and self-treatment as one of several potential remedies to these challenges [4-8]. Most of this research has focused on providing the infrastructure needed to allow patients to self-monitor at home and convey the measurements to caretaking staff in the clinic, focusing on saving transport time for patients, more frequent measurements, and new ways of organizing healthcare institutions $[4,5]$. Other work has investigated patients and healthcare professionals acceptance of these changes in work organizations and at home $[6,7,9]$.

Research within the telemedicine field has suggested meeting these challenges by improving treatment efficiency using both traditional and novel communication technology for remote care and consultations. This includes remote monitoring of patient parameters such as heart rate, blood pressure, weight, oxygen-saturation, medical adherence, and other parameters [10-12]. This allows for monitoring patients in their homes or other unsupervised settings remotely from the treating physician's office, as well as increased patient-empowerment, self-care, and autonomy [5].

However, this alternative paradigm presents several challenges. When a patient is sent home or left unsupervised with a biomedical device. He is no longer personally guided and monitored by a treating physician or nurse, but is left on his own to perform the measurements or take the prescribed medication. As a consequence the quality of the measurement is no longer guaranteed by a healthcare professional, but relies on the patients training and abilities, and on the level of technology support provided. Healthcare professionals thus have to rely on the patient's ability to follow the prescribed treatment plan and guidelines. Medical research has identified that patients to a large extent do not follow such guidelines [1315]. This may result in erroneous and misleading readings, and may lead to the healthcare professional providing a suboptimal treatment due to the low quality of data obtained [16-18].

In order to investigate these challenges, we need methods and tools that will allow us to gain a better understanding of how the patient is acting in situ in the home or unsupervised setting while using pervasive healthcare equipment. For example, we would like to understand to which extent a patient correctly follows the guidelines on how to perform valid home blood pressure measurements. Is the patient sufficiently rested, seated correctly, and in a low-noise environment? Are the data even measured on the right person? Another example could be whether a patient is taking the prescribed medication at the correct time and dose, or if a patient is measuring weight before breakfast, after morning toilet visit, and without clothes as instructed. In other words, contextual factors, that may affect patient adherence and bias measurement data.

Traditional methods useful for this kind of research activities include observations in the home setting by researchers, either directly or through video capture [19]. This requires the researchers to be present in the home and may be very time-consuming and privacy-invading. As such, this method is not feasible for anything more than limited qualitative studies. There is also the risk of inducing bias on patient behavior during measurements, when being observed. Video recordings are less intrusive, but still demand a fairly large technical setup as well as extensive reviewing work. Also, the ability to correctly capture all context information might also be limited when using a single camera only. Is it for example possible to capture video for verifying both whether the user is correctly seated while also monitoring placement of the feet and the handling of the blood pressure device?

As an alternative to direct or indirect observations, it is possible to develop research prototypes that can discretely measure the desired use-context during a healthcare session. 
For example, by developing a prototype with sensors to detect whether the user is correctly seated, and measure noise levels during blood pressure measurement. Rather than using direct observations or video-reviews, the researchers will gain a formalized and discrete data set of relevant user-behavior that can automatically be data-processed and correlated with the measurements. In this way, it may arguably be faster to perform longitudinal studies on a larger population, leading to a more comprehensive understanding than could be achieved with manual observations and data processing.

Creating such prototypes is however a non-trivial task. Depending on the problem under investigation, there is a need for a data acquisition platform, consisting of a computer with relevant processing capabilities, a sufficient communication infrastructure, relevant user interface hardware, video and/or audio recording, patient identification and other relevant features. Often research prototypes are limited to run on a specific device; a smart phone, personal digital assistant, or personal computer platform, and cannot necessarily interoperate with other equipment.

We suggest designing a common and open source software and hardware infrastructure platform for easy development and deployment of pervasive healthcare prototypes with a focus on studying adherence in the unsupervised setting. This platform is mainly intended for exploring the problem domain and providing a design space for constructing and evaluating reliable pervasive healthcare research prototypes. The platform is intended for designing relevant research experiments and studying patients unobtrusively, but could also be used as a platform for pervasive healthcare in the home setting, if adapted and matured as necessary.

Rather than having each research project design and build their own platform from the bottom, we propose having a common platform to start development, allowing reuse of common hardware and software components available for pervasive healthcare and biomedical engineering projects. This could include, but is not limited to research projects exploring: medical adherence issues in the home setting, issues related to healthcare home measurements such as blood pressure, weight, blood sugar, oxygen saturation, and also in the case of rehabilitation programs - the ability of the user to follow the planned exercises in a correct manner. Reusable components could include integration with healthcare devices like blood pressure devices, weight-scales, blood sugar monitors, oxygen saturation monitors, and context-aware sensors such as bed or chair occupancy sensors, movement and activity sensors, but also communication infrastructures could be shared.

In order to identify challenges and define requirements for such a system, we have analyzed a range of pervasive healthcare projects and frameworks.

\section{Analysis of PERVASIVE HeAlthCARE ProJECTS}

Within the last two decades, the ubiquitous and pervasive computing communities have engaged intensively in the healthcare domain, experimenting with novel technology and user interaction techniques for enhancing healthcare. They are inspired by the ubiquitous research tradition as framed by
Weiser [20, 21], with a major focus on the key concepts of calm technology and context awareness for providing a better user experience, and broader access to data and services. Pervasive healthcare has been established as a separate research field within the pervasive computing paradigm $[5,8,22]$, focusing on healthcare in the hospital setting, as well as in the home of the individual patients. Bardram et al. defines it as being "the application of pervasive computing in healthcare" [22] while Varshney defines it as "healthcare to anyone, anytime, and anywhere by removing locational, time and other restraints while increasing both the coverage and the quality of healthcare" [8].

In a recent review, Orwat et al. [4] identified 69 articles describing 67 different systems that investigated the application of pervasive and ubiquitous technology in the healthcare domain. They conclude that a broad and heterogeneous range of systems exist in the field and that these are build and evaluated using a multitude of different technologies. Such technologies include differences in: hardware platform, network and communication protocols, operating system, middleware, programming language, and more. In order for an evaluation platform to support such a diversity of technologies, we need to support a high degree of technology heterogeneity. We thus infer the requirements 1-4 (see section III for list of requirements), with a focus on multi-feature support, heterogeneity, openness, flexibility, and ease-of-use.

Most of the systems investigated by Orwat et al. are only described and evaluated in their prototype stages. Indeed, designing, implementing, and evaluating proof-of-concept prototypes are the preferred methodology of both the pervasive computing and pervasive healthcare communities $[4,5]$. This includes laboratory experiments, followed by possible in situ deployment, e.g. in the home of patients. Orwat et al. conclude that further research on the deployment of pervasive computing systems, including clinical studies, economic and social analyses, user studies, and more, is needed [4]. Reliability issues does not appear to be a major concern, and most often refers to the reliability of devices and infrastructure, rather than the reliability of patients using the equipment in a correct and consistent manner. However, user identification and usagecontext tagging is not ensured in most solutions, prompting for requirements 5-7 to support this in future studies.

Bardram et al. [6] investigates the patient-physician relationship based on the introduction of homecare monitoring equipment, such as a blood pressure device connected to a personal digital assistant, and the implications of these findings for the design of pervasive health monitoring technologies in the home setting. In this study, only limited attention is given to the correct operation by the patient of the blood pressure device, and how this could be quantified and measured, and thus the reliability of the measurements in the home setting is not investigated. We find that support for user-context tagging and identification appears to be relevant for this and related case studies, as stated in requirements 5-7.

Clemensen et al. [23] studies patients suffering from diabetic foot ulcers and how pervasive technologies could assist in improved home care, resulting in fewer visits to the 
hospital. This is achieved by a visiting home nurse using a standard camera phone taking pictures of the ulcer, and conferring with the treating physician at the hospital via phone, data being transferred to a common web platform. This has led to reduced transportation of the patient, and increased cooperation between visiting home nurses and hospital specialists. The project has not allowed the patients increased autonomy, as they must still rely on both a visiting home nurse and teleconference meetings with the hospital physician, and only limited savings of staff time-use. Observations are made qualitatively with researchers interacting with the patients and nurses in the study. There is no means for capturing usage once researchers are not participating. We suggest that requirements 1-8 are relevant for this case study, allowing capture of the use context with a reliable and heterogenic platform.

Grönvall et al. [24] studies how assistive technologies can support home-based rehabilitation for elderly affected by Vestibular dysfunction, a disease which causes dizziness and provokes discomfort and fall situations. But whether the patient is training as instructed cannot be verified by the device, and thus the quality of the rehabilitation is not investigated. Observations are made qualitatively with the researchers being in situ. To promote prolonged usage, we suggest a reliable platform, which could evaluate whether the user complies with the rehabilitation guidelines, and records context-relevant audio and video for researchers to review and evaluate longitudinal usage.

Using pervasive concepts and technologies for improving medical adherence and increasing reliability of self-medication systems have been investigated in a wide range of projects, including the Magic Medical Cabinet [25] and the MedTracker system [26]. Also, Fishkin et al. [27] rely on technology for increasing the reliability of the measurement performed, concerning exactly which medication was taken and when. More recently, Oliveria et al. in the MoviPill project [28] investigated how persuasive technologies could engage the users through adding incentives in the form of competition or similar, to improve their adherence levels, registering when medication is taken, and thus quantifying patient adherence [28]. In these studies, patient adherence to treatment is the main focus, and the quality of the data is investigated as a main objective. Here, patient use-context is partly registered, as the time of taking the medication is considered an important parameter. No thought has been given on sharing a common platform for the technology, and registration of context data is done only in a proprietary fashion. Also here, it would be relevant to record video context and patient identification for evaluating whether the patient is actually taking the medication, also supporting requirements 1-8.

Wagner et al. [15, 29-31] have identified the need for obtaining more reliable home blood pressure measurements than is current clinical practice. Here, it is suggested that a common platform could be employed in order to identify the patient taking the blood pressure, but also the context and circumstances under which the measurement is being performed. The need to detect user stance, activity levels, noise levels, and activities of daily living in general has been identified as important contextual parameters to measure, and provide as meta-data for more valid measurements. It is also discussed that other healthcare related services could benefit from a common platform and open access to context data, and finally, that third party products and services, such as a blood pressure device, should be easy to integrate in a project. Also here, we suggest requirements $1-8$ be facilitated.

In order to allow for experimenting with advanced user interaction and sensor networks, an infrastructure platform is needed. Some infrastructure platforms are based on specialized embedded gateway computers, smart phone based applications, or in the shape of body sensor networks [4, 5, 8, 10]. Also several infrastructure platforms for pervasive healthcare, telemedicine, and ambient assisted living (AAL) projects have been proposed [2, 32-39]. These platforms aim at enabling the patients to monitor themselves at home, employing a range of healthcare devices, and freeing the patients from having to visit a physician. They focus on interoperability of devices, as well as procuring the data transport from sensors to hospitals. We review selected projects in the following.

ElHelw et al. reports on an integrated multi-sensing framework for pervasive healthcare monitoring, as part of the Smart and Aware Pervasive Healthcare Environments (SAPHE) project $[32,33]$. This is a complete and integrated multi-sensing framework for home monitoring of patients, including a sensing platform, data fusion and analysis, and a software architecture suitable for pervasive healthcare applications. System hardware and software implementations have not been made publicly available for further research, and as such, the system does not appear to be extendible with features for investigating reliability and third party sensor nodes.

Eisenhauer has proposed the Hydra Middleware as an open software platform for pervasive healthcare applications [34]. The Hydra Middleware has amongst other been used for implementing an ambient disease management system [35] and as a development platform for integrating wireless devices and sensors into ambient intelligence systems. The software infrastructure has been made available as an open source implementation, but no sensor hardware is available. The platform calls for heterogenic programming language support, and support for multiple concurrent applications and services.

Recently, UniversAAL was introduced as an attempt at creating a unified software platform for ambient assisted living (AAL) projects based on open source [39]. UniversAAL builds on several previous European AAL projects. Like Hydra, UniversAAL is primarily meant as a software and service platform and does not provide specific hardware support for investigations into reliability issues.

The OpenCare project is an open, flexible and easily extendible infrastructure for assisted living and pervasive healthcare solutions [2]. Like UniversAAL and Hydra, no ready-made hardware platform is provided. Instead OpenCare relies on standard commercial off-the-shelf hardware. A long range of commercial and third party sensors have been integrated along with research prototypes to investigate the practical implications of supporting a heterogeneous range of healthcare and assisted living products in a common platform. 
Commercial platforms have been used for telemedicine studies, including the Intel Health Guide PSH6000 (IHG) [36] and the Tunstall mymedic TeleHealth Monitor (TTM) [37]. The IHG and TTM systems have been used in several clinical trials, including in the TELEKAT project [11] to obtain an empirical understanding of the area of telemonitoring, including for chronic patient groups suffering from hypertension, diabetes, asthma, chronic obstructive pulmonary disease (COPD) and others. These platforms use visual and auditory guidance in order to increase the compliance of the patients. However, they do not have the ability to quantify the level of adherence. These platforms are not open, and can as such not be used for doing research on reliable pervasive healthcare and for context tagging of healthcare data.

The reviewed platforms are all based on either a single stationary gateway system with a permanent power supply; either a computer or access point, or a mobile device such as a smart phone. Reliable usage dependents on correct device operation. However, if the hardware platform seizes to operate, infrastructure platforms such as Hydra, UniversAAL, OpenCare, and the Intel Health Guide will simply stop working. This problem does not appear to have been addressed elsewhere in the literature. This could especially be a problem with mobile platforms, which may run out of power if the user is not attentive to this. The permanent power gateways and computers may also fail, as they support limited mobility, usually confined to a room or a building. It could thus be suggested that a reliable infrastructure would support both mobile and fixed installations, the mobile taking over while the user is mobile, and fixed installations being used while user is stationary. Other problems could include network failures. Here, redundancy of network infrastructure could benefit, e.g. support both wired ADSL and $3 \mathrm{G}$ communication. These issues are addressed by requirement 9 .

\section{REQUIREMENTS FOR AN EVALUATION INFRASTRUCTURE}

From the above review of existing pervasive healthcare projects and commercial platforms, we have identified a a range of potential requirements for an evaluation infrastructure prototype for building and evaluating reliable pervasive healthcare projects. The list should neither be considered complete nor universally applicable, but rather an initial starting point for discussing and exploring the possibilities of creating such a platform.

1. Multi-featured Platform: The evaluation infrastructure platform should be able to deliver sufficient computing power to cater to the needs of a wide range of reliable pervasive healthcare research projects - including: situation recognition of context, medical data analysis, user behavior monitoring, and other computational intensive tasks. Also, the platform should be able to support a wide range of communication standards for supporting relevant biomedical and context aware sensors and devices.

2. Heterogeneous Execution Environment: Support for multiple programming languages should be provided by the evaluation platform, allowing researchers to deploy different types of services and applications, and use external frameworks.

3. Openness and Flexibility: The evaluation infrastructure should allow for new frameworks and toolkits to be included, and allow for easy service composition. E.g. the Java Context Awareness Framework (JCAF) [40] could be used for context awareness purposes, while the SPINE framework [41] could be used for user activity tracking and body sensor network integration, and the Hydra, UniversAAL, or OpenCare platform could be used for life cycle management and service composition.

4. Ease-of-use: The evaluation infrastructure should be easy to use and to extend with new features and services for research within reliable pervasive healthcare. Focus should be on the problem under investigation rather than getting the framework to work.

5. Context Recording: Features for audio and video recording of context triggered events should be available. Activating recording e.g. when a user is seated in a chair, a fall is detected, or a user is getting out of bed, etc., so that collected data can be compared to what actually took place for manual verification.

6. User Identification: Easy access to user identification, through both radio based (RFID and NFC) and biometrical (fingerprint, voice, and face recognition) means.

7. User-context Detection: Programmatic access to common movement sensors, chair and bed occupancy sensors etc. should be available.

8. Multi-application Support: In order to support evaluating more than one project on the same platform, e.g. both evaluating for medical adherence, while also evaluating for reliable blood pressure measurement, the evaluation infrastructure platform should support running several evaluation applications concurrently.

9. Reliable Execution Environment: Applications and services running as separate processes should be controlled in a reliable fashion by a life cycle manager, so that services and research applications are kept running and automatically restarted if necessary. Also, the hardware platform should be made reliable, e.g. through redundancy of critical components, as to avoid single points of failure.

\section{SUgGeSted SOLUTION}

We suggest designing a solution that can meet the requirements of section III combining both hardware and software components, and basing it on open source and open hardware, as well as commercial off-the-shelf standard products, rather than proprietary and closed solutions. This would allow the research and commercial community to collaborate in the development and testing of such an 
evaluation infrastructure platform, and ease the reproduction of results.

The suggested solution could be seen as both representing the vision of how a general purpose reliable pervasive healthcare platform might function, as well as being an experimental tool for evaluating infrastructure, sensor-devices and use-context with a focus on reliability and adherence. Thus, the platform could both be useful for creating a complete smart home infrastructure $[42,43]$ supporting ambient assisted living [42], or more special purpose portable telemonitoring health gateway platforms for easy non-stationary deployment in the home of patients, or simply to inform the design and create more reliable stand-alone biomedical devices (see Figure 1).

The suggested solution can never be considered completed, as its open nature allows it to develop over time, and include or replace various components as needed. It is intended to be used for active research with a focus on reliable pervasive healthcare. In this manner, the feasibility prototypes will be deployed in the laboratory as well as in the homes of patients, and the findings from these feasibility case studies will form the empiric background for informing the future design of the solution, while also informing the design of other platforms, such as smart homes or stand-alone biomedical devices.

Requirement 1, 2, 8, and 9 dictates the solution to be heterogeneous and allow different applications and frameworks to co-exist and communicate. This could be achieved by separating the individual services and applications into separate processes, and use inter-process communication. We suggest using standard middleware with adequate tool support, as this will keep productivity high, while allowing for easy integration of new services and components.

We also suggest wrapping biomedical devices and contextaware sensors, as well as general purpose services such as notification, communication, persistence, and other relevant components, into a middleware controlled service layer, governed by a life cycle manager to secure the continued operation of services and applications, as previously suggested in the Hydra, UniversAAL, OpenCare and other service oriented frameworks.

\section{CURRENT RELEI PLATFORM}

The current version of the RELEI platform is an initial implementation of the suggested solution. It allows researchers to utilize the individual components of the platform either as stand-alone components that may easily be integrated into a research prototype, or as part of the OpenCare Service Engine (OCSE) framework. OCSE is used for life cycle management and service composition. Using the OCSE platform, we have explored using middleware to separate processes controlled by the OCSE life cycle manager, meeting the reliability requirements identified (requirement 9). In this way, it has been possible to easily build a number of prototypes investigating various clinical topics of interest using the platform, both in the laboratory and in the home setting. The OCSE platform ensures that a process or service that is failing cannot cause the entire system to fail, and that the process is restarted. This is achieved by separating services and user interface applications by a middleware layer, so that all inter-process communication is done using either socket or named pipes communication. Different versions of OCSE have employed different middleware frameworks. In the two recent versions, the Internet Communication Engine framework (ICE) and Web services using the Windows Communication Foundation framework were used respectively. Initial experiences with this approach appears promising, and it has allowed us to have a platform that is independent of where processes are running, including hardware platforms, as long as they are placed within the same network.

The .NET platform was chosen as the main development platform for RELEI, as this platform can run on several operating systems, including the Windows family, Windows CE, Windows Phone 7, .NET Micro (embedded framework), Linux and Android (via Mono). The choice of middleware has allowed a heterogeneous focus, where external frameworks, toolkits, and applications can easily integrate via the middleware layer using multiple programming languages.

We have created components and service implementations for several typical pervasive healthcare and telemedicine devices, including from A\&D, Omron, Nonin, Corscience, and Beyer. A total of four different commercial blood pressure devices, two ECG devices, three weight-scale devices, two blood sugar devices, and a single pulse/oximetry device. These software components allow researchers easy access to relevant biomedical devices for experimental purposes. Also, contextaware sensors for specialized projects have been developed which are generally applicable in reliable pervasive healthcare research, including: bed-occupancy sensor, movement sensor, door sensor, activity sensor, noise sensor, as well as several prototypes of an intelligent chair and a mobile chair-seat, for measuring whether a patient measuring his blood pressure is correctly seated and in a low noise environment. Also, we have created a medication sensor for tracking medication adherence [44].

We have also created video and audio recording components, which can be triggered by relevant contextual events. Besides such video and audio recordings, the platform can also record the context-sensor data during a healthcare session, allowing for a precise and discrete quantification of user behavior during measurements that can be coupled with the video or audio capture. Previously we have provided research prototypes that use NFC, RFID, and 2D barcode components for identification of users and equipment [45]. We have also integrated face recognition and voice recognition software for user identification. We have interfaced with the SPINE framework [41] for Body Sensor Network support, in order to support fall detection and patient activity tracking.

The RELEI platform (Figure 1) has been implemented and evaluated in three physical versions: One as a stationary smart home base station configuration that we are using for evaluations in our smart home laboratory (Figure 3), another as a brief-case packed portable platform for easy deployment in the home setting of patients (Figure 2). And, finally as a portable tablet-based device. The first two platforms features 
built-in RFID for user identification, web cam and audio, as well as WiFi to $3 \mathrm{G}$ routing facility for ad hoc networking, in order not to rely on the individual patient to provide this functionality.

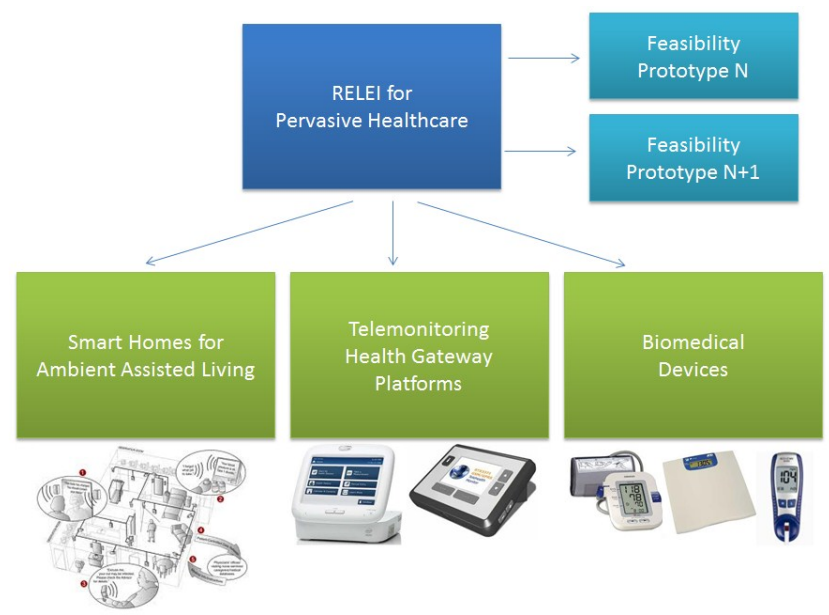

Figure 1. The RELEI platform can be used for assessing the reliability of pervasive healthcare systems. Experiences and components of RELEI may also contribute to the improvement of biomedical devices, telemonitoring gateway platforms and for smart homes.

In Figure 2 the brief case version is displayed attached to one of the reliable home blood pressure measurement evaluation prototypes [30], in order to test for patient stance and noise levels, while measuring a blood pressure.

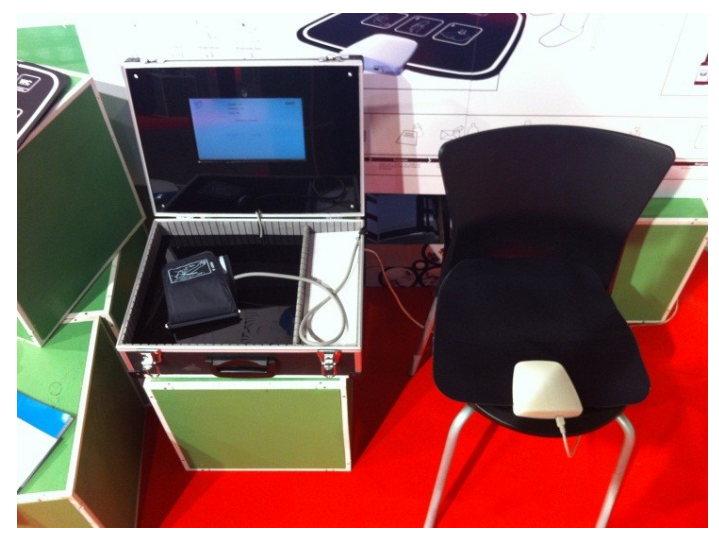

Figure 2. RELEI in the brief case version. Here connected to a blood pressure device and an intelligent chair-seat.

\section{FEASABILITY PROTOTYPES}

Several feasibility prototypes for testing various aspects of reliable pervasive healthcare have been constructed using RELEI both with and without OCSE. This includes three studies investigating different aspects of reliable home blood pressure measurements [29-31]. Also, a feasibility prototype for exploring context-aware medication reminder service as a means for improving medication adherence levels with hypertensive patients has been investigated [44]. A weightscale application have been implemented, testing for patients ability to measure their weight directly after getting out of bed, as recommended for monitoring sudden weight chances.
The latter two prototypes are interfacing with a bedoccupancy sensor to collect knowledge on patient rise times, and a context-aware medication cabinet and an industrial weight scale respectively, for knowledge on when the patient is taking his medication and performing the measurement. The first three constructed prototypes are interfacing with two different chair-occupancy sensors, for investigating patient stance, as well as three different blood pressure measurement devices. One prototype was also integrated with the SPINE framework, to communicate with a wireless body sensor using a TinyOS equipped sensor node, providing an indication of patient activity levels before and during measurements [31].

Using RELEI components we could successfully track patient behavior and context during blood pressure measurements in the unsupervised setting. We recorded audio for verifying whether the patient would speak during measurement, and we recorded whether the user was seated correctly, back supported, and legs not crossed according to guidelines. Using RELEI components enabled us reuse existing components, including the wireless blood pressure device, the audio recording component, as well as the life cycle manager component. Also, the RFID component was used to identify the patients. Another approach could have been to utilize a facerecognition or fingerprint component for this. Also, we used the SPINE component for tracking user activity levels before getting seated, and could update the adherence model with this.

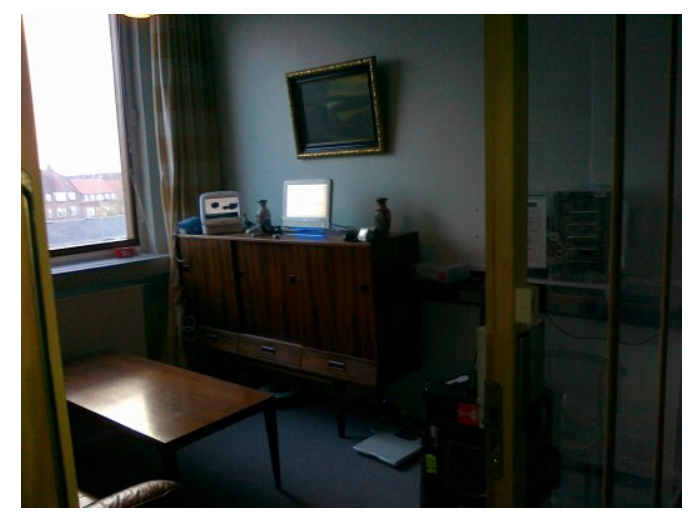

Figure 3. RELEI in the smart home living laboratory (in the center) at the Aarhus University School of Engineering, next to the Intel Health Guide.

In another case study we were also able to successfully track when a patient would leave his bed and when the patient would take the prescribed medication. Again, we used standard components and services, including bed sensor component, medication adherence component, and the notification service component for sending a reminder text message to the patient smartphone, if they had left home without taking the medication.

We are currently investigating using RELEI to model adherence in the unsupervised setting involving various chronic patient groups: COPD, diabetics, hypertensive, Edema, and heart and kidney disease patients in seven research projects. 


\section{DISCUSSION}

Several existing platforms for pervasive healthcare have been presented, both research level, including the SAPHE, UniversAAL, and Hydra platforms, as well as commercial platforms such as the IHG and THM. As such, it could be argued, that there is no need to introduce new platforms. However, while research platforms such as Hydra and UniversAAL are open for extension, they have limited hardware component support out of the box, requiring users to expand the framework with the needed components manually. SAPHE has several biomedical devices supported, but is not available as open source. Also, though Hydra and UniversAAL are based on Java, they remain open to integrate with non-Java applications, due to their reliance on Web services as middleware. On the other hand, the OCSE framework currently used by RELEI is using either ICE or W3C Web services, allowing all frameworks supported by either of these two technologies to integrate. This includes $\mathrm{C}++$, Java, .NET, Python and many other contemporary programming languages. Furthermore, all RELEI components can also be used as standalone components, by linking them directly into evaluation software, independently of the OpenCare platform.

The commercial platforms have a wide range of biomedical devices supported, including weight scales, blood pressure devices, oxygen saturation and blood sugar devices. However, it is not possible to integrate these with context-aware sensors, in order to investigate aspects of reliability. Also, the ability to add external frameworks, such as the SPINE framework or JCAF is not immediately possible in the commercial platforms. The IHG is able to integrate biomedical drivers with members of the Continua Alliance [46]. This is however apparently not targeting user-context tracking sensors and similar devices. As such, it is unclear whether such use of the IHG is feasible for this or not. We argue that it is highly relevant to keep solutions open for modification and integration, rather than closed and inaccessible. This is especially relevant for platforms that should be used for research projects. We also argue that keeping interfaces open, flexible, and simple is highly relevant if we want to make it easy for researches to use the platform. If the interfaces require excessive cognitive activity to master, it might be easier to build a solution from the bottom. Openness and flexibility comes at the price of increased hardware cost and higher power consumption. A mobile device needing to support both GSM/3G communication, WiFi and Bluetooth, would require more advanced hardware components, than a single communication technology device, most likely incurring higher production costs and power usage.

We have so far relied primarily on commercial off-the-shelf hardware platforms and technologies for the RELEI platform, rather than producing dedicated hardware. This could be seen as a weakness, as dedicated hardware is easier to adapt, e.g. with regards to power consumption and communication capabilities. It could as such be beneficial to integrate the periphery hardware elements such as RFID reader, $3 \mathrm{G}$ and WiFi router, and more, into a single unit, along with the main computer and screen. However, we have not chosen this approach in order to maintain a simple platform that is easy and cheap to reproduce, deploy, and test. We argue to wait until the platform has matured sufficiently before producing a dedicated hardware unit. Also, we have not yet fully completed the service layer of the OCSE system, and thus the feasibility prototypes have so far been monolithic. This is however a design choice made within the respective project teams, as OCSE allows the individual application the choice of exposing its interface for use in service composition as needed.

\section{CONCLUSION}

We have analyzed the need for an evaluation platform for testing reliability aspects of pervasive healthcare systems by surveying a range of state-of-the-art pervasive healthcare, biomedical, and telemedicine projects. A list of requirements was provided, and a solution was suggested to meet these requirements. The RELEI platform was presented as an initial, prototypic, and evolutionary platform for exploring aspects of reliability of pervasive healthcare in the home setting.

\section{ACKNOWLEDGMENT}

We would like to thank Martin Kjeldsen, Lars Sarbæk Kristiansen, Anders Udesen Nysom, Ali Rezar Dehdar, and Rene Grønqvist Stenner for programming and testing components of the OCSE and RELEI platforms. Thank you to Troels Skakfjord Magnussen and Jan Harbo Pedersen for working on the SmartHeart feasibility prototype development, and industrial designer Anders Bech Christensen for work on the RELEI brief case version, and SmartHeart prototype. Thank you to Anders Bloch Arnfast for working on the Medication Reminder System. To Jean-Yves Malubungi Mukendi for work on the wired blood pressure device.

\section{REFERENCES}

[1] J. Keye and T. Zitzelberger, "Overview of healthcare, disease, and disability," in Pervasive Computing in Healthcare, Editors: J. Bardram, A. Mihailidis and D. Wan, CRC Press, 2006, pp. 3-20.

[2] S. Wagner and C. Nielsen, "OpenCare project: An open, flexible and easily extendible infrastructure for pervasive healthcare assisted living solutions" in Proceedings of 3rd International Conference on Pervasive Computing Technologies for Healthcare - Pervasive Health, 2009.

[3] Centers for Disease Control and Prevention, National Center for Health Statistics, Health United States. "Limitation of activity caused by selected chronic health conditions among older adults, by age: United States, 2004-2005. Data from the National Health Interview Survey,", Internet: http://www.cdc.gov/nchs/data/hus/hus07.pdf, [Jan. 2011].

[4] C. Orwat, A. Graefe and T. Faulwasser, "Towards pervasive computing in health care - a literature review," BMC Med.Inform.Decis.Mak., vol. 8, pp. 26, Jun 19. 2008.

[5] J.E. Bardram, "Pervasive healthcare as a scientific discipline," Methods Inf.Med., vol. 47, pp. 178-185, 2008.

[6] J. Bardram, C. Bossen and A. Thomsen, "Designing for transformations in collaboration: a study of the deployment of homecare technology", Proceedings of the 2005 international ACM SIGGROUP Conference on Supporting Group Work. 2005. pp 294-303.

[7] [J. Clemensen, S. Larsen and J. Bardram, "Developing Pervasive EHealth for Moving Experts from Hospital to Home," IADIS International Journal of WWW / Internet, vol. II, pp. 57-68, 2005.

[8] U. Varshney, "Pervasive Healthcare and Wireless Health Monitoring," Mobile Networks and Applications, vol. 12, pp. 113-127, 06/01. 2007.

[9] R. Aarhus, E. Grönvall and M. Kyng, "Challenges in participation: Users and their roles in the development of home-based pervasive healthcare applications", Qual Health Res January 2007 vol. 17 no. 1 pp. 122-130. 
[10] A. AbuDagga, H.E. Resnick and M. Alwan, "Impact of blood pressure telemonitoring on hypertension outcomes: a literature review," Telemed.J.E.Health., vol. 16, pp. 830-838, Sep. 2010.

[11] L. Huniche, B. Dinesen, O. Grann, E. Toft and C. Nielsen, "Empowering patients with COPD using Tele-homecare technology," Stud.Health Technol.Inform., vol. 155, pp. 48-54, 2010.

[12] L. Osterberg and T. Blaschke, "Adherence to medication," N.Engl.J.Med., vol. 353, pp. 487-497, 08/04. 2005.

[13] N.R.C. Campbell and D.W. McKay, "Accurate blood pressure measurement: Why does it matter?" Can.Med.Assoc.J., vol. 161, pp. 277-278, 1999.

[14] T.G. Pickering, W.B. White, T.D. Giles, H.R. Black, J.L. Izzo, B.J. Materson, S. Oparil and M.A. Weber, "When and how to use self (home) and ambulatory blood pressure monitoring," J.Am.Soc.Hypertens., vol. 4, pp. 56-61, Mar-Apr. 2010.

[15] S. Wagner, T.S. Toftegaard and O.W. Bertelsen, "Challenges in Blood Pressure Self-Measurement," International Journal of Telemedicine and Applications, 2012.

[16] N.R. Campbell, A. Chockalingam, J.G. Fodor and D.W. McKay, "Accurate, reproducible measurement of blood pressure," CMAJ, vol. 143, pp. 19-24, July 1. 1990.

[17] D.W. McKay, M.K. Raju and N.R. Campbell, "Assessment of blood pressure measuring techniques," Med.Educ., vol. 26, pp. 208-212, May. 1992.

[18] T.G. Pickering, J.E. Hall, L.J. Appel, B.E. Falkner, J. Graves, M.N. Hill, D.W. Jones, T. Kurtz, S.G. Sheps and E.J. Roccella, "Recommendations for blood pressure measurement in humans and experimental animals: part 1: blood pressure measurement in humans: a statement for professionals from the Subcommittee of Professional and Public Education of the American Heart Association Council on High Blood Pressure Research," Circulation, vol. 111, pp. 697-716, Feb 8. 2005.

[19] D. Benyon, Designing interactive systems: a comprehensive guide to HCI and interaction design, Addison Wesley, 2010.

[20] M. Weiser, "The computer for the 21 st century" In Scientific American, September, 1991.

[21] M. Weiser, "Some computer science issues in ubiquitous computing", . In Communications of the ACM, July 36, vol. 7, 1993.

[22] J.E. Bardram, A. Mihailidis and D. Wan, "Pervasive Computing in Healthcare", CRC Press, 2006.

[23] J. Clemensen, S.B. Larsen and N. Ejskjaer, "Telemedical treatment at home of diabetic foot ulcers," J.Telemed.Telecare, vol. 11 Suppl 2, 2005 .

[24] E. Grönvall, R. Aarhus and S.B. Larsen, "Vestibular rehabilitation in the home: design challenges". In the Proceedings of the Ninth Danish HCI Research Symposium 2009, Aarhus, Denmark, 14 December, 2009.

[25] D. Wan, "Magic Medicine Cabinet: A Situated Portal for Consumer Healthcare", in Proceedings of the International Symposium on Handheld and Ubiquitous Computing, 1999.

[26] T.L. Hayes, J.M. Hunt, A. Adami and J.A. Kaye, "An Electronic Pillbox for Continuous Monitoring of Medication Adherence," in Engineering in Medicine and Biology Society, 2006. EMBS '06. 28th Annual International Conference of the IEEE, pp. 6400-6403, 2006.

[27] K. Fishkin, M. Wang and G. Borriello, "A ubiquitous system for medication monitoring", in Proceedings of the 2nd international conference on pervasive computing, 2004.

[28] R. de Oliveira, M. Cherubini and N. Oliver, "MoviPill: improving medication compliance for elders using a mobile persuasive social game," in Proceedings of the 12th ACM international conference on Ubiquitous computing, pp. 251-260, 2010.

[29] S. Wagner, T. Toftegaard and O. Bertelsen, "Context Assessment during Blood Pressure Self-measurement Utilizing the Sensor Chair," Ambient Intelligence, pp. 295-299, 2011.

[30] S. Wagner, T.S. Toftegaard and O.W. Bertelsen, "Increased Data Quality in Home Blood Pressure Monitoring through Context Awareness," in Pervasive Computing Technologies for Healthcare (PervasiveHealth), 20115 th International Conference on, pp. 1-4, 2011.
[31] S. Wagner, T.S. Toftegaard and O.W. Bertelsen, "Novel approach for ensuring increased validity in home blood pressure monitoring" in Proceedings of the 4th International Conference on Pervasive Computing Technologies for Healthcare, 2010, pp. 1-4, 2010.

[32] M. ElHelw, J. Pansiot, D. McIlwraith, R. Ali, B. Lo and L. Atallah,". An integrated multi-sensing framework for pervasive healthcare monitoring" in Proceedings of the 3rd International Conference on Pervasive Computing Technologies for Healthcare, 2009.

[33] SAPHE, "Architecture Overview", Internet: http://ubimon.doc.ic.ac.uk/ saphe/m686.html, [Jan. 2011].

[34] [34] M. Eisenhauer, P. Rosengren and P. Antolin, "HYDRA: a development platform for integrating wireless devices and sensors into ambient intelligence systems," in Internet of Things, New York, NY: Springer Verlag, 2010, pp. 367-373.

[35] [H. Eikerling, G. Gräfe, F. Röhr and W. Schneider, "Ambient healthcare systemsusing the hydra embedded middleware for implementing an ambient disease management system". In Proceedings of the Second International Conference on Health Informatics, pages 82-89, Portugal, 2009

[36] Intel Corporation, "Intel Health Guide PHS6000", Internet: http://www.intel.com/corporate/healthcare/emea/eng/healthguide/pdfs/H ealth_Guide_Product_Brief.pdf[Jan 2011].

[37] Tunstall Limited, "Telehealth solutions." Internet: http://www. tunstall. co.uk/Our-products/Telehealth-solutions [Jan. 2011]

[38] A. Bamis, D. Lymberopoulos, T. Teixeira and A. Savvides, "Towards precision monitoring of elders for providing assistive services," in Proceedings of the 1 st international conference on Pervasive Technologies Related to Assistive Environments, pp. 49:1-49:8, 2008.

[39] S. Hanke, C. Mayer, O. Hoeftberger, H. Boos, R. Wichert, M.R. Tazari, P. Wolf and F. Furfari, "universAAL-An Open and Consolidated AAL Platform," in Ambient assisted living: 4. AAL-Kongress 2011, Berlin, Germany, January 25-26, 2011, pp. 127, 2011.

[40] J.E. Bardram, "The Java Context Awareness Framework (JCAF) - a service infrastructure and programming framework for context-aware applications," Pervasive Computing, pp. 98-115, 2005.

[41] R. Gravina, A. Guerrieri, G. Fortino, F. Bellifemine, R. Giannantonio and M. Sgroi, "Development of Body Sensor Network applications using SPINE," in Systems, Man and Cybernetics, 2008. SMC 2008. IEEE International Conference on, pp. 2810, 2008.

[42] G. Demiris, "Smart homes and ambient assisted living in an aging society. New opportunities and challenges for biomedical informatics," Methods Inf.Med., vol. 47, pp. 56-57, 2008.

[43] International Medical Informatics Association (IMIA). Working Group on Smart Homes and Ambient Assisted Living. Definition. Internet: http://www.health-smarthomes.org/index.html [Jan, 2011]

[44] S. Wagner, A.B. Arnfast, T.S. Toftegaard and O.W. Bertelsen, "Medication Reminder System using Calm Guidance," in Proceedings of the 11th Danish Human-Computer Interaction Research Symposium (DHRS2011), 2011.

[45] N.B. Christensen and S. Wagner, "Using near field communication technology to achieve near-zero-configuration of sensors," in Proceedings of the 5th Annual International Conference on Mobile and Ubiquitous Systems: Computing, Networking, and Services, pp. 38, 2008 .

[46] R. Carroll, R. Cnossen, M. Schnell and D. Simons, "Continua: An interoperable personal healthcare ecosystem," IEEE Pervasive Computing, pp. 90-94, 2007. 